УДК 338.2

\title{
А. А. Алексеев
}

Уральский государственный аграрный университет, Екатеринбург, e-mail: break-fast@mail.ru

\section{Т. И. Кружкова}

Уральский государственный аграрный университет, Екатеринбург, e-mail: rustale@yandex.ru

\section{А. В. Ручкин}

Уральский государственный аграрный университет, Екатеринбург, e-mail: alexeyruchkin87@gmail.com

\section{О. А. Рущицкая}

Уральский государственный аграрный университет, Екатеринбург, e-mail: olgaru-arbitr@mail.ru

\section{О. Е. Рущицкая}

Уральский государственный аграрный университет, Екатеринбург, e-mail: ruscha@yandex.ru

\section{ОТ ИНТЕРНЕТА ВЕЩЕЙ К ИНТЕРНЕТУ ПОВЕДЕНИЯ: ГОСУДАРСТВЕННОЕ РЕГУЛИРОВАНИЕ ЦИФРОВОЙ ТРАНСФОРМАЦИИ АПК}

Ключевые слова: интернет поведения, интернет вещей, сельское хозяйство, агропромышленный комплекс, государственное регулирование, цифровая экономика.

Одним из результатов цифровой трансформации российского агропромышленного комплекса стало массовое применение участниками рынка технологий интернета вещей. Следующим этапом развития становится переход к новой, ещё более перспективной концепции - интернету поведения. В статье авторы рассматривают концептуальные и специфические отличия интернета вещей от интернета поведения, на кейсах демонстрируют и выявляют специфику развития данного направления в сельском хозяйстве. Особое внимание уделяется взаимодействию субъектов в ходе осуществления хозяйственной деятельности, возможностям влияния и переходу к субъект-объектной парадигме. В связи с масштабностью процесса и его значением для национальной экономики возникает проблема его государственного регулирования в интересах устранения этих рисков. Предлагаются принципы регулирующего воздействия государства для снижения рисков, а также основные факторы модели для повышения эффективности и снижения технических и административных барьеров, возможных для сельского хозяйства. Определен технологический минимум, позволяющий говорить о необходимости установления регулирующего воздействия со стороны государственных структур. Данная модель направлена на решение не только существующих проблем хозяйствующих субъектов, но и социально-экономических, и экологических проблем.

\section{A. A. Alekseyev}

Ural State Agrarian University, Yekaterinburg, e-mail: break-fast@mail.ru

\section{T. I. Kruzhkova}

Ural State Agrarian University, Yekaterinburg, e-mail: rustale@yandex.ru

\section{A. V. Ruchkin}

Ural State Agrarian University, Yekaterinburg, e-mail: alexeyruchkin87@gmail.com

O. A. Ruschitskaya

Ural State Agrarian University, Yekaterinburg, e-mail: olgaru-arbitr@mail.ru

O. E. Ruschitskaya

Ural State Agrarian University, Yekaterinburg, e-mail: ruscha@yandex.ru

\section{FROM THE INTERNET OF THINGS TO THE INTERNET OF BEHAVIOR: STATE REGULATION OF THE DIGITAL TRANSFORMATION OF THE AGRO-INDUSTRIAL COMPLEX}

Keywords: Internet of behavior, Internet of things, agriculture, agro-industrial complex, state regulation, digital economy. 
One of the results of the digital transformation of the Russian agro-industrial complex was the mass use of Internet of Things technologies by market participants. The next stage of development is the transition to a new, even more promising concept - the Internet of behavior. In the article, the authors consider the conceptual and specific differences between the Internet of things and the Internet of behavior, demonstrate and identify the specifics of the development of this direction in agriculture. Special attention is paid to the interaction of subjects in the course of economic activity, the possibilities of influence and the transition to the subject-object paradigm. Due to the scale of the process and its importance for the national economy, there is a problem of its state regulation in the interests of eliminating these risks. The principles of the regulatory impact of the state to reduce risks are proposed, as well as the main factors of the model to increase efficiency and reduce technical and administrative barriers possible for agriculture. The technological minimum is defined, which allows us to talk about the need to establish a regulatory impact on the part of state structures. This model is aimed at solving not only the existing problems of economic entities, but also socio-economic and environmental problems.

\section{Введение}

Понятие «интернет поведения» вошло в научный оборот в 2012 году. Его автором является Гот Найман (Gote Nyman), профессор психологии Хельсинкского Университета. Он предположил, что на основании данных, считываемых с человека, можно предсказать его дальнейшее поведение. В своей работе обращался к психологическим и этическим последствиям такого анализа [1]. В широкий научный оборот термин «интернет поведения» был введён на Gartner IT Symposium / Хро 2020 Americas (Стамфорд, штат Коннектикут, США) 19 октября 2020 года. Аналитики компании Гартнер (Gartner) объявили интернет поведения главным технологическим трендом 2021 года. Они прогнозируют, что к концу 2025 года более половины населения мира будет участвовать как минимум в одной программе IoB [2].

Интернет поведения является логическим развитием концепции интернета вещей (Internet of Things, IoT), предполагающей исключить участие человека в процессах взаимодействия физических объектов («Things») между собой и с внешним миром. Под интернетом («Internet») понимается не конкретная глобальная сеть Интернет (т.е. построенная на базе протоколов ТСР/ IP), а любая сеть («Web»), соответствующая технологическим требованиям. Такая терминологическая неточность объясняется случайностью, точнее, тем, что именно так его назвал Кевин Эштон, соучредитель и исполнительный директор исследовательской группы в области сетевой радиочастотной идентификации Auto-ID Labs. В 1999 году он опубликовал статью «Эта штука «Интернет вещей»» [3]. Интернет вещей включает в себя технологии подключения к сети (сотовая и спутниковая связь, wi-fi и др.), оборудование (сенсоры, системы хранения данных, аппаратные устройства безопас- ности), программное обеспечение и соответствующую инфраструктуру. Поскольку прародителем интернета вещей является концепция межмашинного взаимодействия (Machine-to-Machine, M2M), то её также можно считать предшественником интернета поведения.

Одним из направлений исследования интернета вещей является измерение степени влияния искусственного интеллекта на поведение людей. Исследования показали, что большинство сотрудников западных компаний считают, что искусственный интеллект - более объективный и эффективный руководитель, чем человек [4]. Джош Берсин, один из ведущих мировых исследователей в области управления талантам, считает, что системы управления кадрами, управляемые искусственным интеллектом, позволят существенно сократить расходы на управление кадрами. Искусственный интеллект принимает решения не интуитивно, а на основе анализа фактов, что делает его более объективным [5].

В то же время исследования учёных из Саутгемптонского университета показали, что алгоритмы могут побуждать людей к более рисковому поведению и принятию значительно более рискованных решений, нежели обычно. Доктор Янив Ханох (Yaniv Hanoch), доцент кафедры управления рисками Саутгемптонского университета, опубликовал результаты исследования влияния цифровых ассистентов на поведение человека. Это влияние оказалось гораздо больше, чем предполагалось ранее, и при желании способствовать принятию человеком неоправданных, высоко рисковых решений [6].

Таким образом, исследователи, изучающие интернет поведения, описывают его как логическое развитие идеи интернета вещей. Поскольку интернет поведения способен повлиять на поведение живых существ, в пер- 
вую очередь человека, ряд исследователей обнаруживают связанные с этим этические проблемы $[1,6]$. Но большая часть исследователей описывает достоинства интернета вещей, к которым относят ускорение обмена информацией и принятия решений, и обусловленное этим снижение затрат $[2,5,7]$.

Поскольку интернет поведения постепенно переходит из состояния концепции в реально функционирующую систему, а количество подключенных к сети объектов постоянно растёт [2], возникает объективная необходимость изучения возможности государственной поддержки перехода от интернета вещей к интернету поведения в хозяйственной деятельности субъектов агропромышленного комплекса Российской Федерации.

Объектом наблюдения данного исследования выступают организации агропромышленного комплекса, использующие технологии интернета вещей и интернета поведения.

Объектом исследования является механизм государственного регулирования агропромышленного комплекса в части его цифровой трансформации.

Выбор объекта исследования был обусловлен несколькими объективными факторами. Необходимость цифровой трансформации экономики стала постулатом государственной политики. По мнению авторов, достигнутые позитивные результаты не являются основанием для снижения темпов развития. Применение по-настоящему новых технологии должно происходить при участии государства, которое, в обмен на оказание мер поддержки, получает право на регулирование всего процесса. Ограниченность ресурсов требует не просто концентрации усилий на отдельных направлениях, но и создания стандартов, при участии субъектов агропромышленного комплекса. Субъективным фактором выбора объекта исследования является многолетняя работа авторов над проблемами государственного регулирования сельского хозяйства и подготовки высококвалифицированных кадров для агропромышленного комплекса.

Предметом исследования выступают социально-экономические отношения, возникающие в процессе государственного регулирующего воздействия на субъектов - организации агропромышленного комплекса, использующие технологии интернета вещей и интернета поведения.
Цель исследования заключается в определении основных направлений и методов государственного регулирования агропромышленного комплекса в интересах ускорения перехода от интернета вещей к интернету поведения.

В соответствии с целью были поставлены и реализованы следующие задачи, определившие логику и структуру исследования:

- дать авторское определение понятию «интернет поведения в сельском хозяйстве», основываясь на существующих взглядах на интернет вещей;

- изучить кейсы применения интернета поведения в агропромышленном комплексе различных стран мира;

- описать достоинства и недостатки интернета поведения, имеющие значения для обоснования выбора форм и методов регулирования;

- установить принципиальную возможность воздействия государства на переход от интернета вещей к интернету поведения в агропромышленном комплексе;

- определить базовые принципы и практические методы регулирования.

\section{Материал и методы исследования}

Под «интернетом поведения» (Internet of Behaviors, IoB) в данной статье понимается система передачи и обработки данных, считываемых с человека или любого другого живого организма, причём этот процесс происходит постоянно, в обе стороны, в режиме реального времени. Такая система может не только считывать и анализировать большой поток данных (big data), но и управлять принятием решений. Существует несколько концепций интернета поведения. Единой технологической платформы не существует, но ведётся множество перспективных разработок в данном направлении (искусственный интеллект, чипирование, система социального кредита, телемедицина и многие другие).

Наибольший научный интерес для авторов вызывает возможность влияния государства, то есть оказания регулирующего воздействия, на процесс перехода от интернета вещей к интернету поведения в целях ускорения этого процесса.

В данном исследовании использован ряд методов научного познания. Теоретический анализ показал разнородность подходов к определению интернета поведения. Сравнение как метод познания позволило выявить схожие с интернетом поведения 
технологические концепции. Использование метода научной аналогии заключалось в перенесении существующих механизмов государственного регулирования агропромышленного комплекса на процесс перехода от интернета вещей к интернету поведения. Системный подход был использован при установлении взаимосвязи развития цифровой экономики и фудшеринга. Метод кейсов позволил изучить практический опыт применения интернета поведения в различных странах мира. Принцип сочетания исторического и логического в их единстве позволил взглянуть на переход к интернету поведения как на этап процесса цифровой трансформации глобальной экономики, и логика развития которого свидетельствует о необходимости участия государства в этом процессе.

Теоретическую и методологическую основу исследования составляют фундаментальные положения экономической теории по государственному регулированию экономики, научные публикации российских и зарубежных учёных по вопросам применения интернета вещей и интернета поведения, статистические данные, полученные в результате ранее проведённых исследований.

\section{Результаты исследования и их обсуждение}

Научной проблемой является отсутствие определения понятия «интернет поведения в сельском хозяйстве». Это связано с тем, что, в отличие от понятий «интернет вещей» (IoT) и «интернет вещей в сельском хозяйстве» (IoTAg), не существует строгого научного определения понятия «интернет поведения» (IoB) [8]. Чаще всего интернет поведения рассматривается как результат эволюции интернета вещей, его более высокая ступень. В результате исследования авторы пришли к выводу, что проблема детерминирования «интернета поведения в сельском хозяйстве» (IoBAg) вызвана разнообразием подходов (взглядов) на данный феномен маркетинговых, психологических, технологических, философских и других. Изучив опыт применения понятия «интернет вещей в сельском хозяйстве», мы пришли к выводу, что оно имеет два основных, принципиально различающихся значения:

- интернет поведения в сельском хозяйстве - это концепция, предполагающая управление работниками сельхозпредприятий, сельскохозяйственными животными и растениями искусственным интеллектом;
- интернет поведения в сельском хозяйстве - это совокупность технологий, позволяющих собирать массив данных с сельскохозяйственных животных и растений, а также работников сельхозпредприятий, обрабатывать эти данные, и влиять на поведение (состояние) живых организмов с помощью подключенных в единую сеть устройств.

Интернет поведения в сельском хозяйстве как концепция не предполагает конкретного материального воплощения, а подразумевает, что автоматизированное управление сельским хозяйством, основанное на обработке огромного объёма информации и множестве типовых сценариев, более эффективно и оперативно, чем классический менеджмент. Технологии интернета поведения в сельском хозяйстве, наоборот, имеют конкретную вещественную (датчик, механизм) или информационную (программный продукт или набор сценариев) форму, которая предназначена для решения конкретной практической задачи. Оба указанных определения подразумевают, что принятие управленческого решения и его исполнения возможны без участия человека.

Другой научной проблемой является отсутствие описания, в чём именно заключаются достоинства и недостатки интернета поведения, в чём различие между интернетом поведения и интернетом вещей. По нашему мнению, ключевыми критериями различия в данном случае выступают субъектность и объектность. В интернете вещей объектами взаимодействия выступают вещи, то есть предметы неживой природы, обладающие изменяющимися характеристиками. Субъектами является сенсоры, сканеры, передатчики и другие считывающие и передающие информацию устройства. Взаимодействие между ними происходит в виде передачи данных о состоянии объекта или в виде команд на изменение этого состояния. Человек (или любое другое живое существо) из этого процесса исключаются. В интернете поведения объектами являются живые организмы люди, животные и растения. В интернете вещей факт воздействия субъекта на объект очевиден и не вызывает возражений. В интернете поведения субъект может управлять объектом, обладающим свободой воли, и данное обстоятельство ставит под вопрос допустимость самого феномена интернета поведения. Для того, чтобы выявить достоинства и недостатки интернета поведения, рассмотрим его практическое применение на примере кейсов из различных отраслей АПК. 
Японская корпорация Fujitsu разработала систему GyuHo SaaS (яп. «шагающая корова»), состоящую из носимого датчика для коров, программного обеспечения для обработки данных и их передачи на смартфон или компьютер. На основании полученных данных можно корректировать кормление, доение и сон животных, обнаруживать заболевания на ранней стадии, и, самое важное, вычислить благоприятный период для зачатия. В результате, успешность искусственного оплодотворения с $44 \%$ вырастает до 90\%. К системе подключено около 40 тысяч коров [9].

В Массачусетском технологическом институте (МIT) ведётся разработка электронных компонентов и систем для растений в рамках проекта «растительная нанобионика». В результате экспериментов учёные смогли считать данные о наличии определённых химических веществ в почве с шпината. Корни шпината передали биосигнал листьям. Этот сигнал считала инфракрасная камера и передала его на компьютер по беспроводной сети. В перспективе такая технология позволит собирать данные о предстоящих изменениях климата и возможных экологических проблемах [10].

В Норвегии разработана и применяется на практике система распознавания рыб на фермах. Для выявления заражённых паразитами лососей, рыб сканируют сенсора-

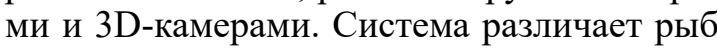

на основе уникальных пятен вокруг глаз, рта и жабр, каждая из попавших в объектив особей получает собственную виртуальную медицинскую карточку. Если какая-то особь заболевает, её отлавливают, и отправляют в карантин. По оценкам специалистов, выявление заболевания на ранней стадии позволит сократить смертность примерно на $50-75 \%$ [11].

В Российской Федерации функционирует Национальная система учета и регистрации животных. Её основой является программный пакет REGAGRO, с помощью которого проводится регистрация и идентификация животных, ведётся их учёт и формируется всё необходимая документация. Система также способна отслеживать состояние здоровья животных, их перемещение, смену собственника, обеспечить взаимодействие между владельцами животных и ветеринарными организациями. Для идентификации животных используется компактный сканер, совместимый со смартфоном или планшетом [12]. Другим перспективным направлением использования интернета поведения в АПК признано измерение психофизического состояния оператора сельхозтехники [13].

Основываясь на перечисленных кейcax, и исходя из содержания самого понятия, рассмотрим позитивные и негативные аспекты применения интернета поведения в сельском хозяйстве. Для удобства сопоставления представим их в виде таблицы.

Позитивные и негативные аспекты применения интернета поведения в сельском хозяйстве

\begin{tabular}{|c|l|c|l|}
\hline № & \multicolumn{1}{|c|}{ Позитивные аспекты } & № & \multicolumn{1}{|c|}{ Негативные аспекты } \\
\hline 1 & $\begin{array}{l}\text { Повышает качество жизни в сельской местности, } \\
\text { поскольку открывает доступ к другим технологиям } \\
\text { (например, интернет поведения в медицине) }\end{array}$ & 1 & $\begin{array}{l}\text { Требует высокоразвитой цифровой ин- } \\
\text { фраструктуры на больших территориях } \\
\text { с низкой плотностью населения }\end{array}$ \\
\hline 2 & $\begin{array}{l}\text { Создаёт привлекательные для «продвинутых» мо- } \\
\text { лодых специалистов рабочие места, в т.ч. в сельской } \\
\text { местности }\end{array}$ & 2 & $\begin{array}{l}\text { Необходимы специалисты соответству- } \\
\text { ющей квалификации, способные посто- } \\
\text { янно совершенствовать свои навыки }\end{array}$ \\
\hline 3 & $\begin{array}{l}\text { Продолжает глобальный тренд (интернет вещей), что } \\
\text { гарантирует дальнейшее технологическое развитие }\end{array}$ & 3 & $\begin{array}{l}\text { Существует множество технологиче- } \\
\text { ских платформ, часто несовместимых } \\
\text { между собой }\end{array}$ \\
\hline 4 & $\begin{array}{l}\text { Позволяет собирать и обрабатывать огромный мас- } \\
\text { сив данных, часть которых ранее была недоступна }\end{array}$ & 4 & $\begin{array}{l}\text { Возможен несанкционированный доступ } \\
\text { кданным, их искажение или хищение }\end{array}$ \\
\hline 5 & $\begin{array}{l}\text { Обеспечивает рост производительности труда и } \\
\text { снижение издержек на единицу продукции }\end{array}$ & 5 & $\begin{array}{l}\text { Высокая цена ошибки, обусловленная } \\
\text { взаимодействием с живыми организмами }\end{array}$ \\
\hline 6 & $\begin{array}{l}\text { Преимущественно интенсивный способ развития } \\
\text { бизнеса, уместный в условиях ограниченности пло- } \\
\text { щадей или воздействия на окружающую среду }\end{array}$ & $\begin{array}{l}\text { В отдельных регионах возможно оттор- } \\
\text { жение интернета поведения по этическим } \\
\text { (елигиозным, культурным) причинам }\end{array}$ \\
\hline 7 & $\begin{array}{l}\text { Способствует созданию положительного образа } \\
\text { агропредприятия и росту его капитализации }\end{array}$ & 7 & $\begin{array}{l}\text { Необходимо изменить или отменить } \\
\text { множество нормативно-правовых актов }\end{array}$ \\
\hline
\end{tabular}

Составлено авторами на основании кейсов $[9,10,11,12,13]$ 
Таким образом, применение интернета поведения не лишено недостатков, но обладает и очевидными достоинствами. Часть проблем, усложняющих этот процесс, во многом совпадают с общими проблемами цифровой трансформации АПК, которые подробно описаны российскими учёными [14].

Позитивный экономический эффект от перехода к интернету поведения очевиден. Применение технологий интернета поведения способствует увеличению объёмов производства, снижению издержек на единицу продукции, стимулирует развитие смежных отраслей и цифровой инфраструктуры. Для максимизации позитивного эффекта, по мнению авторов, необходимо участие государства в процессе перехода от интернета вещей к интернету поведения.

\section{Заключение}

В результате нашего исследования была получена модель государственного регулирующего воздействия на переход от интернета вещей к интернету поведения в агропромышленном комплексе. Субъектом в данной модели выступает организация агропромышленного комплекса, использующая определённые технологии, такие как системы точного земледелия, IP-камеры, умные метеостанции, системы доения животных, RFID-метки интегрированные системы управления, и т.д., все вместе, или по отдельности. Существует технологический минимум, который может быть охарактеризован как минимально необходимый функционал цифровой инфраструктуры - скорость передачи данных, количество одновременно подключенных устройств, пространство для локального и облачного хранения больших данных, доступ к серверам онлайн-сервисов, расположенных за пределами государства. Субъект совершенствует свою деятельность, применяя новые технологии интернета вещей и накапливая всё возрастающий объём больших данных. Его производственно-сбытовые возможности возрастают (по сравнению с организациями, не использующими возможности интернета вещей), но отстают от мировых лидеров в отрасли. Руководство субъекта понимает, что сможет некоторое время сохранить лидерство на местном рынке только благодаря протекционизму государства (например, заградительным импортным пошлинам). На свободном рынке такой субъект проиграет рыночное соревнование. Это является критической угрозой существования субъекта. Угроза может быть устранена путём технологического усовершенствования субъекта, заключающегося в дополнении технологий интернета вещей ещё более прогрессивными технологиями интернета поведения. Государство является интересантом эффективного, соответствующего самым современным стандартам сельского хозяйства, поскольку оно гарантирует обеспечение продовольственной безопасности страны. Поэтому оно готово оказывать регулирующее воздействие на процесс развития своего агропромышленного комплекса.

Регулирующее воздействие государства также должно широко затрагивать информационно-образовательную среду, от расширения глоссария ведомственного проекта «Цифровое сельское хозяйство» [15] до корректировки федеральных государственных образовательных стандартов в части формируемых компетенций.

Работа модели заключается в изменении качественных характеристик субъекта, которое происходит в результате применения технологий интернета поведения под влиянием инструментария государственной поддержки. В результате субъект получает доступ к новым технологиям, компенсацию части издержек и более совершенную инфраструктуру. Для наглядности представим работу модели графически.

Гипотеза исследования предполагает, что данная модель является принципиально оптимизируемой в результате применения регулирующего воздействия со стороны государства. Возможные отклонения в работе модели обусловлены ранее описанными в нашей работе рисками. Концепция перехода от интернета вещей к интернету поведения в агропромышленном комплексе, по мнению авторов, должна базироваться на принципах незыблемости рыночных механизмов ценообразования продукции АПК, стимулированию перехода от простых цифровых инструментов к более сложным, развитию высокотехнологичной инфраструктуры в сельской местности. 


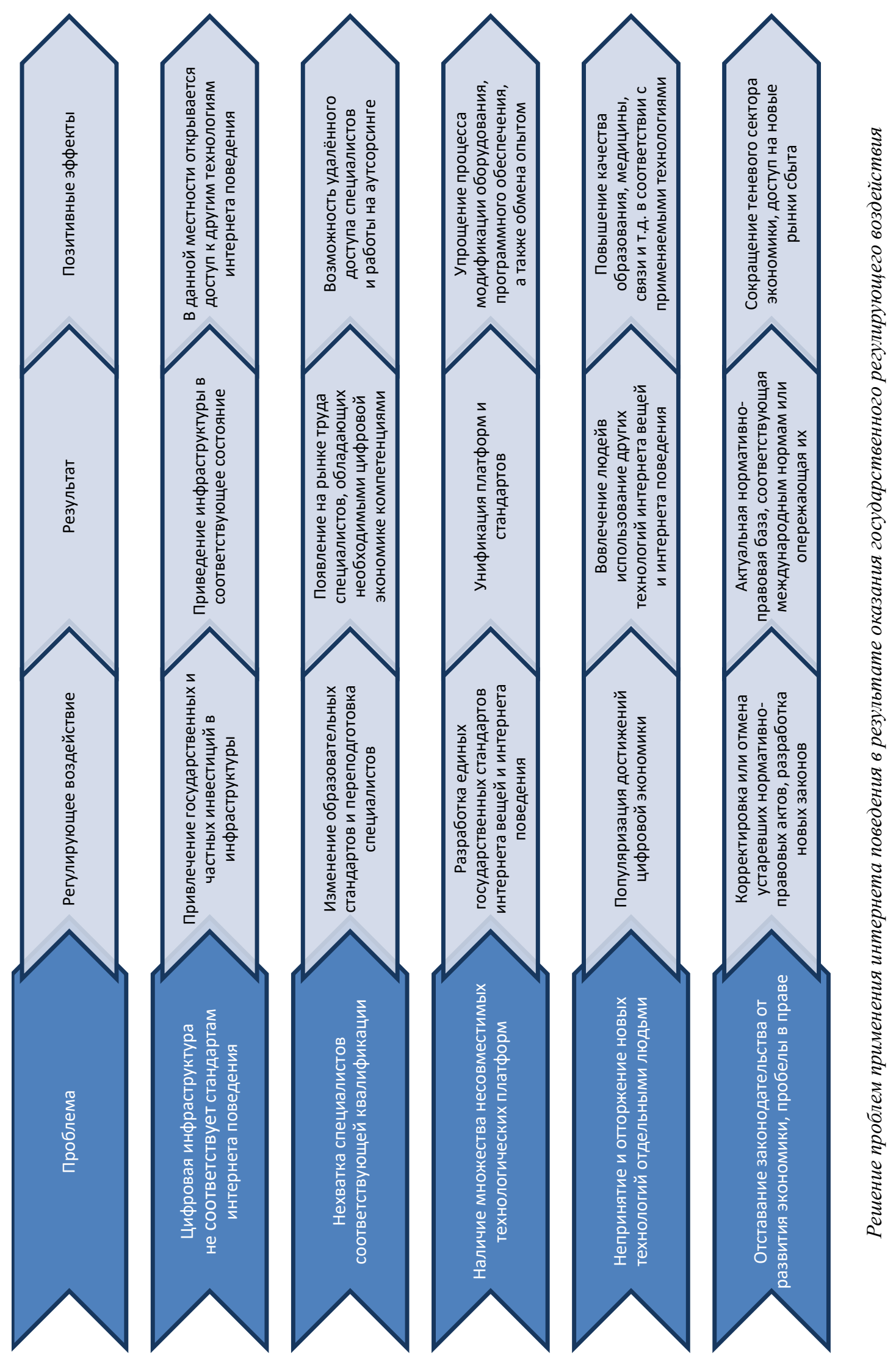

ВЕСТНИК АЛТАЙСКОЙ АКАДЕМИИ ЭКОНОМИКИ И ПРАВА №6 2021 
Прежде чем предлагать конкретные формы и методы регулирования, установим базовые принципы регулирования:

- очевидным результатом регулирования должно быть снижение издержек на единицу продукции;

- стимулирование перехода от простых цифровых инструментов к более сложным ориентировано на повышение производительности труда, а не улучшения PRобраза организации;

- развитие высокотехнологичной инфраструктуры в сельской местности не должно ограничиваться густонаселёнными и экономически развитыми районами;

- недопустима дискриминация субъектов предпринимательской деятельности получателей государственной поддержки по признаку вовлеченности в процесс цифровой трансформации;

- регулирующее воздействие должно учитывать социальные и экологические факторы в целях недопущения социальных конфликтов, аналогичных кейсам «шихана Куштау» в Башкирии или «войной с вышками 5G» в Дагестане.

В результате изучения работы построенной модели был установлен ряд действий, направленных на оптимизацию работы модели на базе вышеперечисленных принципов и с учётом потенциальных рисков:

- активная разъяснительная работа, заключающаяся в доведении до потенциальных пользователей всех преимуществ интернета поведения;

- создание единых технологических стандартов, унификация уже существующих технологий и платформ;

- обеспечение конфиденциальности и целостности обрабатываемых данных, защите от несанкционированного доступа;

- совершенствование законодательства, подразумевающее отмену препятствующих развитию интернета поведения правовых норм;

- подготовка компетентных специалистов, причём не только инженеров, но и менеджеров;

- привлечение к инвестированию в соответствующую инфраструктуру частных инвесторов;
- субсидирование перехода от интернета вещей к интернету поведения в агропромышленном комплексе;

Особо отметим, что проблемы, связанные с переходом от интернета вещей к интернету поведения в агропромышленном комплексе, не могут быть решены административно-командными мерами. Невозможно (и не нужно) заставить фермера использовать описанные технологии, если он сам этого не хочет или не может. Это необходимо учитывать при определении основных направлений регулирования. По нашему мнению, к таким направлениям регулирования относятся поддержка малого и среднего бизнеса, стимулирование молодёжного предпринимательства, устранение правовых и технологических ограничений, развитие цифровой инфраструктуры. В рамках указанных направлений следует прибегнуть к конкретным методам регулирования:

- обеспечение доступа к сервисам обработки и хранения больших данных, получаемых от сельхозтоваропроизводителей;

- государственный заказ на разработку российского программного обеспечения, соответствующего требованиям защиты информации;

- разработка программ повышения квалификации работников АПК в целях формирования у них необходимых компетенций, финансирование привлечения к проведению занятий ведущих специалистов;

- компенсация части расходов на закупку оборудования и программных пакетов отечественного производства.

Перечисленные методы регулирования способствуют переходу российского АПК на качественно новый уровень, тем самым способствуя решению целого ряда экономических, социальных и экологических проблем. В результате исследования авторы пришли к выводу, что переход на технологии интернета поведения в хозяйственной деятельности российских агропредприятий является сложной и важной организационно-экономической задачей, решение которой требует активного участия государства. 


\section{Библиографический список}

1. Gote Nyman The psychology behind Internet of Behaviors (IB). 4 October 2012 [Электронный ресурс]. URL: https:/gotepoem.wordpress.com/2012/10/04/the-psychology-behind-internet-of-behaviors-ib/ (дата обращения: 06.04.2021).

2. Gartner Identifies the Top Strategic Technology Trends for 2021. STAMFORD, Conn., 19 October 2020. [Электронный ресурс]. URL: https://www.gartner.com/en/newsroom/press-releases/2020-10-19-gartner-identifies-the-top-strategic-technology-trends-for-2021 (дата обращения: 04.04.2021).

3. Ashton, K. (22 June 2009). “That 'Internet of Things' Thing”. [Электронный pecypc]. URL: http://www. rfidjournal.com/articles/view?4986 (дата обращения: 05.04.2021).

4. Аргументы недели [Электронный ресурс]. URL: https://argumenti.ru/world/2021/02/707834 (дата обращения: 03.04.2021).

5. AI in HR: A Real Killer App BY JOSH BERSIN [Электронный ресурc]. URL: https://joshbersin. com/2018/06/ai-in-hr-a-real-killer-app/ (дата обращения: 06.04.2021).

6. Yaniv Hanoch, Francesco Arvizzigno, Daniel Hernandez García, Sue Denham, Tony Belpaeme, and Michaela Gummerum // Cyberpsychology, Behavior, and Social Networking. 2020. Vol. 24. № 5. [Электронный pecypc]. URL: https://www.liebertpub.com/doi/full/10.1089/cyber.2020.0148 (дата обращения: 06.04.2021).

7. What Is the "Internet of Things"? By Eric Escobar on 29 April 2015 [Электронный pecypc]. URL: https:// www.scientificamerican.com/article/what-is-the-internet-of-things/ (дата обращения: 06.04.2021).

8. The Industrial Internet Consortium's Vocabulary Task Group [Электронный ресурc]. URL: https://www. iotone.com/terms/glossary/alphabet/i (дата обращения 06.04.2021).

9. Матюшкина М. Коров кормят цифрами: интернет вещей добрался до сельского хозяйства [Электронный ресурc]. URL: https://www.dp.ru/a/2020/08/21/Korov_kormjat_ciframi (дата обращения: 05.04.2021).

10. Wong M.H., Giraldo J.P., Kwak S.-Y., Koman V.B., Sinclair R., Lew T.T.S., Bisker G., Liu P., Strano M.S. Nitroaromatic detection and infrared communication from wild-type plants using plant nanobionics // Nature Materials. 2017. № 16. P. 264-272. DOI: 10.1038/nmat4771.

11. МТС Медиа. Умное сельское хозяйство: четыре примера из разных стран [Электронный ресурс]. URL: https://media.mts.ru/business/175807/ (дата обращения: 05.04.2021).

12. Национальная автоматизированная система регистрации, учёта и идентификации животных REGAGRO [Электронный ресурс]. URL: http://regagro.ru/opisanie/obscee-opisanie (дата обращения: 04.04.2021).

13. Искусственный интеллект даст плоды в сфере АПК [Электронный ресурс]. URL: https://dataeconomy.ru/tpost/llixjjkab1-iskusstvennii-intellekt-dast-plodi-v-sfe (дата обращения: 07.04.2021).

14. Voronin B.A., Mitin A.N., Pichugin O.A. The complexity of digitization of agriculture in Russia. Proceedings of the International Scientific and Practical Conference «Advances in Intelligent Systems Research». 2019. DOI: 10.2991/ispc-19.2019.29.

15. Ведомственный проект «Цифровое сельское хозяйство». [Электронный ресурc]. URL: http://mcx. ru/upload/iblock/900/900863 fae06c026826a9ee43e124d058.pdf (дата обращения: 07.04.2021). 